\title{
Ontology-informed Lattice Reduction Using the Discrimination Power Index
}

DOI:

10.1007/978-3-030-23182-8_12

\section{Document Version}

Accepted author manuscript

Link to publication record in Manchester Research Explorer

\section{Citation for published version (APA):}

Quboa, Q., Behnaz, A., Mehandjiev, N., \& Rabhi, F. (2019). Ontology-informed Lattice Reduction Using the Discrimination Power Index. In 24th International Conference on Conceptual Structures (ICCS2019) Springer Nature. https://doi.org/10.1007/978-3-030-23182-8_12

\section{Published in:}

24th International Conference on Conceptual Structures (ICCS2019)

\section{Citing this paper}

Please note that where the full-text provided on Manchester Research Explorer is the Author Accepted Manuscript or Proof version this may differ from the final Published version. If citing, it is advised that you check and use the publisher's definitive version.

\section{General rights}

Copyright and moral rights for the publications made accessible in the Research Explorer are retained by the authors and/or other copyright owners and it is a condition of accessing publications that users recognise and abide by the legal requirements associated with these rights.

\section{Takedown policy}

If you believe that this document breaches copyright please refer to the University of Manchester's Takedown Procedures [http://man.ac.uk/04Y6Bo] or contact uml.scholarlycommunications@manchester.ac.uk providing relevant details, so we can investigate your claim.

\section{OPEN ACCESS}




\title{
Ontology-informed Lattice Reduction Using the Discrimination Power Index
}

\author{
Qudamah Quboa $^{1}$, Ali Behnaz ${ }^{2}$, Nikolay Mehandjiev ${ }^{1}$, and Fethi Rabhi ${ }^{2}$ \\ ${ }^{1}$ Alliance Manchester Business School, University of Manchester, Manchester, UK \\ ${ }^{2}$ School of Computer Science and Engineering, University of New South Wales, Australia \\ \{qudamah.quboa, n.mehandjiev\}@manchester.ac.uk, \\ \{ali.behnaz,f.rabhi\}@unsw.edu.au
}

\begin{abstract}
The increasing reliance on data for decision making has led to a number of techniques for automatic knowledge acquisition such as Formal Concept Analysis (FCA). FCA creates a lattice comprising partial order relationships between sets of object instances in a domain (extent) and their properties (intent). This is mapped onto a semantic knowledge structure comprising domain concepts with their instances and properties. However, this automatic extraction of structure from a large number of instances usually leads to a lattice which is too complex for practical use. Algorithms to reduce the lattice exist. However, these mainly rely on the lattice structure and are agnostic about any prior knowledge about the domain. In contrast, this paper uses existing domain knowledge encoded in a semantic ontology and a novel relevance index to inform the reduction process. We demonstrate the utility of the proposed approach, achieving a significant reduction of lattice nodes, even when the ontology only provides partial coverage of the domain of interest.
\end{abstract}

Keywords: FCA, Semantic structures, Lattice reduction.

\section{Introduction}

Three main factors underpin the growth of data gathered by organizations today: the low costs of storage, the increasing role of ICT in business and the reliance on data for decision making at all organizational levels. Only a small percentage of the data gathered is ever used, though, because of the difficulties in automatically analyzing the meaning (semantics) of the data. The problem of knowledge acquisition [1] includes processing voluminous data [2], understanding its meaning and relationships [3], and then presenting the results using simplified and relevant models [4].

Manual semantic tagging of data for the purpose of knowledge acquisition is too labor intensive for practical use, and researchers are trying to automate this. One such technique is Formal Concept Analysis (FCA). FCA takes a table of incidence relations between sampled data instances and their properties, called a formal context, and constructs a lattice of partial order relationships between the instance sets and between the property sets. However the lattices generated by FCA are too complex for practical semantic analysis of real-world datasets. Existing approaches to reduce these lattices 
are based on mathematical measurements of relevancy [4] and do not consider existing knowledge of the targeted domain, even when it is formalized and represented in a semantic structure or an ontology.

The similarities between FCA and ontology-based semantic representations have inspired a number of approaches drawing on both, such as merging different ontologies[5,6], ontology modelling and attribute exploration $[7,8]$. However, the use of existing semantic knowledge encoded as ontology to support the lattice reduction has not been explored until now. Our main research question is thus:

How can we use domain ontology to support attaching semantics to further instances in the domain through FCA?

In this paper, we present an approach which answers these questions by using prior domain knowledge (encoded in semantic ontology format) to classify and guide the reduction process of a sampled formal context where not all instances are in the ontology. The approach extracts semantic structures from the ontology, transforms them into a formal context and aligns the two formal contexts using two main activities (1) basic mapping using known instances which exist in both the ontology and the sampled formal context; and (2) advanced alignment combining similarity measurements that use both instances and properties of the sampled formal context plus the concepts added from the ontology. Finally, the proposed approach uses the ontology generated one to reduce the sampled one, resulting in a lattice output of FCA which we show to be significantly simpler than the lattice produced without the approach, and where concepts in the lattice are aligned with ontology concepts from the targeted ontology, thus classifying previously unclassified instances from the sample domain. The approach relies on a new concept relevancy metric called Discrimination Power Index $(D P I)$.

The proposed approach enables the construction of intelligent analysis tools that could be integrated within information retrieval and knowledge processing systems, by addressing the problem of large lattices produced when applying FCA to real-world data. We present results from a realistic case study which confirm the feasibility and the validity of the proposed approach.

The remainder of this paper is structured as follows: Section 2 describes the background of the work. Sections 3 and 4 describe the proposal and define the proposed similarity and reduction indices and mechanisms. Section 5 provides a case study to evaluate the proposed approach and Section 6 concludes the paper.

\section{Background}

\subsection{Formal Concept Analysis (FCA)}

Formal Concept Analysis (FCA) is a mathematical mechanism to automatically analyze the structure of a target domain [2,9] and create a lattice representing partial order relationships between sets of observed (sampled) object instances in a domain of interest and between sets of their properties constructed from a matrix of incidence relationships. The resultant lattice is used to reason with these relationships and map them 
onto a semantic knowledge structure representing concepts in the domain with their instances, properties and specialization relationships [2].

Definition 1. (Formal Context) A formal context is a triple $K:=(G, M, I)$, where $G$ and $M$ are two sets of instances and properties respectively and $I$ is a set of relations between instances of $G$ and properties of $M, I \subseteq G \times M$.

Definition 2. (Formal Concept) A formal concept is a pair $(A, B)$, such that $A \subseteq G$, $B \subseteq M$, where $A=B^{\prime}\left(B^{\prime}=\{g \in G \mid \forall m \in B:(g, m) \in I\}\right) \quad$ and $B=A^{\prime}\left(A^{\prime}=\{m \in M \mid \forall g \in A:(g, m) \in I\}\right)$.

The set of instances $A$ represents the extension of the formal concept (extent) and the set of properties $B$ represents the intent of the formal concept (intent).

Definition 3. (Subconcept and Superconcept) The partial ordering relationship $(\leq)$ for context $K$ may exist among each of the formal concepts. It is defined by a subset relation between the concepts' extents or the superset relation between the concepts' intents. That is $\left(A_{1}, B_{1}\right) \leq\left(A_{2}, B_{2}\right) \Leftrightarrow A_{1} \subseteq A_{2}\left(\Leftrightarrow B_{2} \subseteq B_{1}\right)$ where the concept $\left(A_{1}, B_{1}\right)$ is considered a more specific concept, a subconcept of $\left(A_{2}, B_{2}\right)$, compared to $\left(A_{2}, B_{2}\right)$, which is a superconcept of $\left(\mathrm{A}_{1}, \mathrm{~B}_{1}\right)$.

Definition 4. (Concept Lattice) A concept lattice $(\Lambda)$ is the set of all formal concepts ordered by their partial ordering relationship $(\leq)$ of the context $K$ and formulated as $\Lambda=(K, \leq)$.

\subsection{Semantic Web Ontology}

Ontology is defined as an explicit representation of a formal conceptualization of a specific domain where the semantics of information is specified using human-readable text formulated using a machine-readable language $[10,11]$ such as the Web Ontology Language (OWL).

Domain experts have developed standardised and general-purpose ontologies to capture knowledge and share information in multiple disciplines such as the National Center for Biomedical Ontology [12], the Financial Industry Business Ontology (FIBO) [13], and the Institutional ontology [14]. Most often, domain ontologies are designed to determine a set of data and its structure for the use of other programs. Ontology can be used as a data sharing mechanism between different software agents or applications such as the use of the ontology as a data interchange format [15] and as a data integration mechanism [16].

\subsection{Existing Lattice Reduction Techniques}

A major issue with FCA is the large size of lattices generated from realistic data sets because of exceptions and noise [2, 17]. A number of algorithms have been developed to reduce the lattice based on the structure of the lattice itself. These are based on redundant information removal, simplification or selection [4].

Redundant information removal techniques aim to remove instances and/or properties without affecting the general lattice structure. Examples include incomplete decision contexts reduction [18] and attribute reduction by deletion transformations [19]. 
Simplification reduction is any technique that focuses on creating a high-level abstraction of the formal concept lattice, which only keeps the main key aspects. Examples are "junction based on instances similarity" (JBOS) reduction [20], and Fuzzy KMeans clustering reduction [21].

Selection reduction represents any technique that focuses on choosing specific instances, properties and/or concepts according to their relevancy measurements (a set of constraints that needs to be satisfied). Selection reduction can be based on different factors, such as weight (frequent weighted concept reduction [22]), logic (based on user's attributes priorities [23]), or hierarchies (based on hierarchically ordered attributes [24]). This type of reduction is applied after the construction of the formal context has been completed [4].

All these techniques share a common shortfall by failing to consider existing domain knowledge, making the results more vulnerable to systemic noise in the data.

\subsection{Integrating FCA and Ontologies}

The definitions of FCA and ontology-based domain modelling may create a wrong impression that both approaches are similar, whereas the FCA approach is quite different from the ontology approach in terms of core perspectives and language representations. Indeed, FCA follows an "inductive" approach, which is focused on constructing concepts from individuals (extensional description of the domain), and represents intents and extents by atomic level of representation. Ontologies follow the opposite "deductive" approach, which is focused on inferring and reasoning relations between concepts starting from the concepts themselves (intensional definition of concepts), and representing them using a richer expression representation language [3, 25]. Therefore, Ontologies and FCA could complement each other allowing better understanding of, and dealing with the complexity of data and knowledge [3].

This complementarity has motivated research proposals for integrating these techniques. An example is the use of FCA to merge different ontologies based on a bottomup approach with the help of natural language processing (NLP) and selective documents from the domain of interest $[5,6]$. Another example is the use of an FCA approach in ontology modelling and the exploration of properties to discover implications over the target context [7,8]. Yet, none of the proposed approaches uses domain knowledge (represented in a semantic ontology) in the reduction process of the FCA.

\subsection{Similarity Measurements}

We use three similarity measurements to align the two different formal contexts (ontology-derived and sampled) and integrate them. The first two are well-known:

Jaccard similarity coefficient index. This well-known similarity measurement [26] is based on the following formula:

$$
S_{J a c}=\frac{\left|B_{1} \cap B_{2}\right|}{\left|B_{1} \cup B_{2}\right|}
$$


The Jaccard index of any two instances is the ratio of the number of common properties divided by the number of all properties that belong to at least one instance.

Hamming Distance index. This is also a well-known similarity measurement [27] and is based on the following formula:

$$
D_{\text {hamming }}=b+c
$$

where $\mathrm{b}$ represents the number of properties that do not belong to the concept 1 but exist in the concept 2, while c represents the number of properties that belong to the concept 1 and do not exist in the concept 2.

Both indices treat all properties as of equal importance whilst our observations demonstrate that some properties have a higher discriminatory power than others. We thus introduce a new complementary index called Discrimination Power Index (DPI), described below.

\section{Ontology-Informed Lattice Reduction Approach}

Our approach uses existing knowledge about the domain to simplify the lattice $\Lambda$ when extracting structure from sampled data represented in a formal context $K$. We assume that the existing knowledge is in the ontology $\Omega$ and is encoded in OWL.

The proposed approach for the FCA reduction could be divided into four main steps (shown in Fig. 1): (a) Data Extraction: extracting relevant portion from the ontologybased knowledge base; (b) Data Transformation: transforming the ontology data and structure into a formal context format; (c) Data Alignment: aligning it with the sampled formal context; and (d) Data Reduction: reducing the unnecessary complexity of the formal context through the ontology concepts using a combination of measurements that allows a simplified and more stable FCA lattice to be produced.

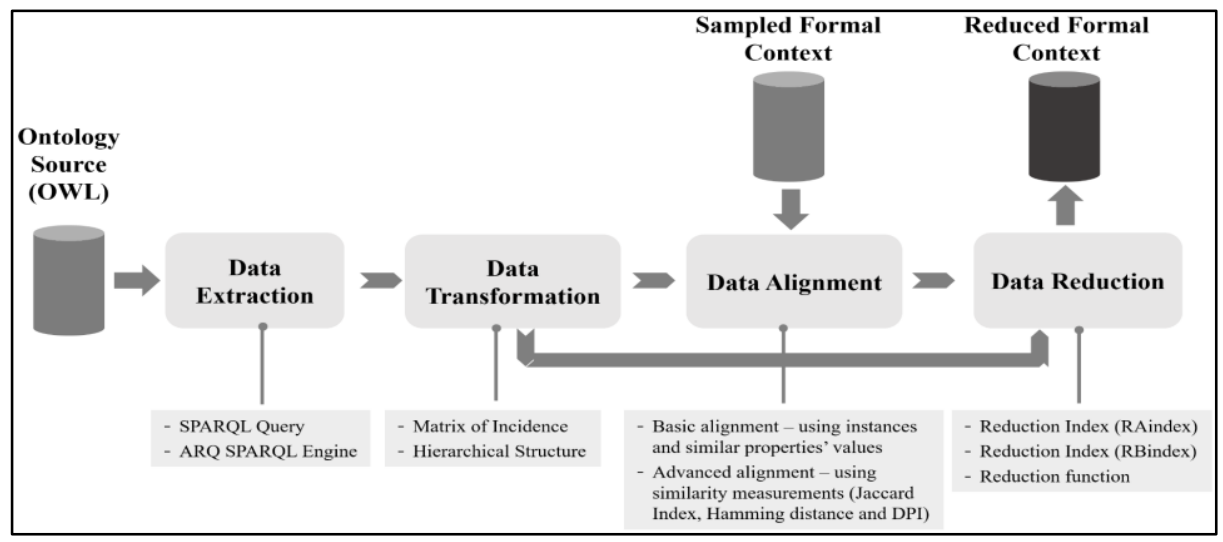

Fig. 1. The general outline of the Ontology-informed Lattice Reduction approach.

During the initial processing steps (a) and (b) of the proposed approach, data from the semantic ontology source is extracted (using SPARQL queries) and transformed into 
an acceptable formal context format (matrix of incidence). This creates the baseline for the integration between the ontology data and the targeted FCA lattice. It allows the alignment to be carried out as well as the enrichment of the targeted FCA lattice with high-level structural concepts (from the ontology).

The Data Alignment step (c) starts by basic mapping - identifying the set of instances $G_{\Omega}$ that exist in both $\Omega$ and in $K$, using them to identify and mark their corresponding ontology concepts from $\Omega$ in $K$, adding the properties from these ontology concepts to $G_{\Omega}$, thus extending the current $K$ into $K_{e}$. The approach then automatically associates the remaining instances $G_{R}=G \backslash G_{\Omega}$ that exist only in $K$ to ontology concepts from $K_{e}$ using:

(1) The properties they have in common with the shared instances $G_{\Omega}$ using the Jaccard Similarity index and Hamming Distance index (see Section 2.5), and

(2) The discrimination power of their properties using DPI (see Section 4.1).

The approach is thus an example of the selection reduction techniques according to Dias and Vieira [4].

This is followed by step (d) where $K_{e}$ is reduced using two reduction measurements (RAindex and RBindex) with their evaluation functions and the reduction function (see Section 4.2). RAindex aims to keep any property that is essential to a specific ontology concept from the used ontology and flag any property that has an uncertainty in the decision. RBindex is a complementary measurement to ensure the right reduction decision is applied for the properties flagged as uncertain by RAindex. Finally, reduction function is applied to the flagged incidence relationships (resulted from applying RAindex and RBindex).

The resultant formal context $K_{R}$ has three notable features:

- It is smaller than the original context and has a simpler lattice.

- It depends on the existing domain knowledge as captured in the ontology $\Omega$. This is unique amongst the existing alternative reduction approaches.

- The reduced lattice $\Lambda$ is similar to, but not identical to the structure of the ontology $\Omega$ due to the existence of extra properties and instances.

It is worth mentioning that

(1) The idea behind the Data Extraction and the Data Transformation are indeed similar to the work presented by Andrews and Polovina [28] specifically in the transformation of the extracted triples into binary relationships. However, our approach differs as follows:

(a) Our extraction approach works with ontologies and uses standardized semantic query language (SPARQL) as well as standardized query engine (ARQ Engine). This allows us to apply the approach to different ontologies (encoded in various semantic formats) and increases the usability and the replications of the work in a wide range of development environments.

(b) The outcome of the transformation stage is different as the extracted information is transformed into (i) matrix of incidence relationships as well as (ii) its hierarchical structure.

(c) The technical implementations of those two stages are different because of the formats of the data and the way the data is being used. 
(2) The approach uses the hierarchical structure from the ontology and set of properties and incidence relationships' values to align and simplify the formal context. This is specifically applied to all the proposed measurements of the approach (DPI, RAindex and RBindex).

\section{Formal Description of Data Alignment and Reduction}

\subsection{Data Alignment}

To assign an instance from the sampled formal context to one of the existing ontology concepts, a combination of different similarity indices is used to evaluate and align concepts from both contexts based on their intents and extents. In this step, a Jaccard similarity coefficient index is first used. If this fails to produce a single "best match", the Hamming distance is used, and if that is still inconclusive with several candidate concepts for best match, we use our proposed index DPI.

$D P I$ is thus a complementary metric. It selects one of the candidate ontology concepts based on the discrimination power of their properties. The fewer the instances that share a certain property, the higher the discrimination power of this property:

$$
D P I=\frac{\left|\left\{\forall g \in G \mid b \in B_{1} \cap B_{2}:(g, b) \in I\right\}\right|}{|G|}
$$

In this equation, $B_{1}$ is the set of properties of the evaluated unclassified instance, and $B_{2}$ is the set of properties filtered by previous similarity indices for the same instance (defining the intent of candidate ontology concepts). DPI then is the ratio of the number of all instances that have incidence relationships with both $B_{1}$ and $B_{2}$ and the complete set of instances. This index relies on the sampled formal context $K$ (using both $G$ and $M)$ to avoid misclassifying or choosing the wrong ontology concept during the transform and align steps of the approach in an attempt to consider the big picture during the calculation.

The assessment of different instances during the alignment process is always comparing any unknown instance with already assigned instances (aligned with the concepts from the used ontology) and this applies to all the used indices in this step. The result of this step is an extended formal context incorporating both the sampled data and the data extracted from the knowledge base.

\subsection{Data Reduction}

During this step, the extended formal context $\left(K_{\text {new }}:=\left(G_{\text {new }}, M_{\text {new }}, I_{\text {new }}\right)\right)$, which results from the alignment process above, is passed to the reduction process where two indices are applied before applying the reduction function. These indices identify the noise and the complexity that can be removed without affecting the stability of the sampled formal context by utilizing the knowledge from the hierarchical structure of the ontology and the formal context derived from it. The ontology guides the reduction procedure and works as a reference point at all times. 
The first index (RAindex): this is proposed to evaluate the value of each property regarding each one of the concepts from the domain ontology. This is to provide an indication whether a property is essential to a specific concept from the ontology or if there is an uncertainty in the decision.

To do that, we scan every property from the extended formal context to check (1) whether all its instances have the evaluated classification concept from the ontology (meaning it is essential) or (2) whether none of its instances have the evaluated classification ontology concept (meaning it can be removed). This process is applied for each one of the ontology concepts and is formally represented as follows:

$$
\begin{gathered}
\text { RAindex }=\frac{\left|\left\{\forall g \in G_{\text {new }} \mid \exists m \in M_{\text {new }} \cap M \wedge c \in M_{\text {new }} \cap M_{\text {Ontology }}:(g, m) \in I_{\text {new }} \cap(g, c) \in I_{\text {new }}\right\}\right|}{\left|\left\{\forall \in G_{\text {new }} \mid \exists m \in M_{\text {new }} \cap M:(g, m) \in I_{\text {new }}\right\}\right|} \\
f(\text { RAindex })= \begin{cases}S t o p & \text { if }(\text { RAindex })=0 \text { or } 1 \\
\text { RBindex } & \text { otherwise }\end{cases}
\end{gathered}
$$

Equation (4) takes the number of all instances that have incidence relationships with both the target concept (the evaluated classification concept from the ontology) and a property that exists in both the initial formal context and the new extended formal context. This is divided by the number of the instances that have an incidence relation with the current evaluated property (regardless of their classification concepts from the ontology). The calculation of RAindex starts from the most abstracted concept of the ontology (the top level of the extracted hierarchical structure of the used ontology) to the most specialized concepts (the bottom level of the hierarchical structure).

Then the ratio of these two parts (RAindex) is evaluated based on its value (5). If the $R A$ index value is 1 , this means the evaluated property is a key property of the ontology concept or if the value is 0 then there are no incidence relationships connecting the evaluated property and the ontology concept. In both cases, there is no need for any reduction to apply. Otherwise, the second reduction index (RBindex) is applied to make the right judgment.

The second reduction index (RBindex). This is a complementary index to ensure that the right action is applied, which allows the reduction process to be completed. It provides an indication where the reduction is necessary by flagging both the property and the ontology's concept that needs the reduction. This is based on a specific reduction percentage (provided by the user of the system).

To do so, we evaluate the instances of the selected property (received from RAindex) that share the targeted concept from the ontology with all the instances of the same ontology concept and check whether the ratio passes the reduction threshold or not. If the case is the latter, then this is finished by flagging the unnecessary incidence relationships within the selected property (which will be removed without damaging the stability of the formal context). This is formally defined as follows:

$$
\text { RBindex }=\frac{\left|\left\{\forall g \in G_{\text {new }} \mid \exists m \in M_{\text {new }} \cap M \wedge c \in M_{\text {new }} \cap M_{\text {Ontology }}:(g, m) \in I_{\text {new }} \cap(g, c) \in I_{\text {new }}\right\}\right|}{\left|\left\{\forall g \in G_{\text {new }} \mid c \in M_{\text {new }} \cap M_{\text {Ontology }}:(g, c) \in I_{\text {new }}\right\}\right|}
$$




$$
f(\text { RBindex })=\left\{\begin{array}{cc}
\text { Trim }(m, c) & \text { if }(\text { RBindex })<\text { Reduction Percentage } \\
\text { Stop } & \text { otherwise }
\end{array}\right.
$$

Equation 6 presents the second reduction index as the ratio of the dividend part of (4) (the same translation provided in RAindex section) and the total number of instances which have incidence relationships with the evaluated ontology's concept (regardless of their evaluated property from the formal context). If the ratio of the index (7) is over the reduction threshold then the evaluated property is an important property and should not be reduced. Otherwise, this indicates that the evaluated property related to the current ontology concept can be removed.

Based on the results of both RAindex and RBindex, the reduction function is applied when needed. Its function is to remove the incidence relationships between the instances and the evaluated property related to the used ontology concept. This is done by scanning the extended formal context for any flagged incident relationship (resulted from RAindex and RBindex) and removing them from there. The formula of this function is as follows:

$$
\begin{aligned}
& \operatorname{Reduction}(m, c)=\forall g \in G_{\text {new }} \mid m \in M_{\text {new }} \cap M \wedge c \in M_{\text {new }} \cap \\
& M_{\text {Ontology }} \wedge(g, m) \in I_{\text {new }} \wedge(g, c) \in I_{\text {new }} \Rightarrow(g, m)=0
\end{aligned}
$$

It is worth mentioning that (1) the sequence of selecting the ontology's concepts to apply the reduction indices is based on the level of the concepts in the original ontology. The reduction procedure is starting from level 0 which is the most general ontology concept, moving down level by level towards the most specific ontology concepts. (2) Both indices are relying on the intents and extents of the new formal context, the ontology structure and incidence relationships to apply the right reduction at right places and avoid causing instability in the evaluated context. (3) The reduction is not a blind reduction and provides enough precautions and considerations before removing any incidence relationships between the evaluated property and its instances that could cause instability to the whole process. (4) The result is resistant to wide-spread (systematic) noise as reduction indices rely on existing domain ontology to guide the reduction.

\section{Applying the Approach to Machine Learning Ontology and Formal Context}

This section tests the effectiveness of the approach by applying it to a case study of machine learning. Indeed, machine learning and predictive analytics techniques have become increasingly popular with the proliferation of data, enabling value to be extracted from data which is too plentiful for human analysis.

Designing and implementing a predictive analytics study is a complex process requiring business understanding, data understanding, data preparation, modelling, evaluation and deployment [29]. Modelling focuses on selecting the appropriate machine learning technique and adjusting parameters to generate correct results. The decision to use a specific technique is driven by the characteristics of the data and the objectives of 
the study. However, even an experienced data scientist can lack the holistic knowledge about all possible modelling techniques, and no single modelling technique has absolute merit over others [30].

In this case study, we use semantic technology to capture and represent the knowledge related to machine learning techniques [31,32] to support model selection. The knowledge is extracted and represented in an ontology covering a set of popular machine learning techniques, built into Microsoft Azure Machine Learning. This is an integrated analytics solution which enables a data scientist to select and deploy machine learning techniques on the cloud [33]. We have adopted the selection logic suggested by Azure Machine Learning to determine the appropriate machine learning techniques for different situations, and to construct our ontology (see Fig. 2).

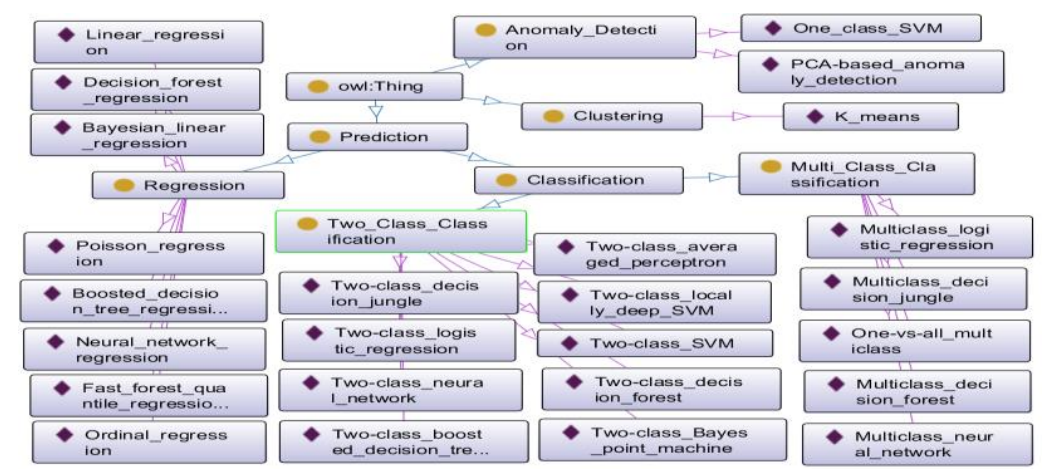

Fig. 2. The Machine Learning Techniques ontology (adapted from [33]).

To illustrate the workings of the approach given the space constraints of this paper, we first use a simplified set of sampled data as in the formal context presented in Table 1. We then apply the proposed approach. Fig. 3 shows the lattice of the extended formal context resulting from aligning the ontology with the sampled data (Data Alignment step). Then Fig. 4 shows the reduced lattice based on the reduced formal context (Data reduction step).

Some properties in Table 1 such as 'Fast training time' and 'Unsupervised learning' are evidently not essential for the majority of the techniques. Our approach has detected that and applied the reduction process to these properties. Other properties, such as 'Find unusual occurrences' and 'Discover structure' are not popular in the formal context (only appear in a couple of techniques), but are important for differentiating their instances, as they refer to two main machine learning categories: anomaly detection and clustering respectively (see Fig. 4), and should not be reduced (and the system has identified that).

In the smaller example from the first phase, the calculated similarity is $100 \%$. This is a very good indication as it reflects how the properties of the resultant lattice are related to the concepts from the ontology. When we apply the same calculation to the larger dataset in the second phase, the similarity is some $85 \%$. This is expected since many properties and instances of the sampled formal context are not found in the ontology. 
Furthermore, the pre-reduction lattice has 18 unique nodes of which only 10 are left after applying the reduction approach, a reduction of $44.44 \%$. This result is with an RBindex threshold of $40 \%$. Different thresholds were tested, for example at $30 \%$ the reduction was $16.66 \%$, while increasing the threshold above $40 \%$ did not achieve significant improvement of the reduction effect so $40 \%$ seems optimal here.

Table 1. The Machine Learning Techniques formal context (adapted from [33]).

\begin{tabular}{|c|c|c|c|c|c|c|c|c|}
\hline & $\begin{array}{l}\text { Predict } \\
\text { values }\end{array}$ & $\begin{array}{c}\text { Find } \\
\text { unusual } \\
\text { occurrences }\end{array}$ & $\begin{array}{l}\text { Discover } \\
\text { structure }\end{array}$ & \begin{tabular}{|c|} 
Predict \\
between \\
two \\
categories \\
\end{tabular} & \begin{tabular}{|c|} 
Predict \\
between \\
several \\
categories
\end{tabular} & $\begin{array}{c}\text { Supervised } \\
\text { learning }\end{array}$ & $\begin{array}{c}\text { Unsupervised } \\
\text { learning }\end{array}$ & $\begin{array}{c}\text { Fast } \\
\text { training } \\
\text { time }\end{array}$ \\
\hline Ordinal_regression & $x$ & & & & & $x$ & & \\
\hline Poisson_regression & $x$ & & & & & $x$ & & \\
\hline $\begin{array}{l}\text { Fast_forest_quantile_regress } \\
\text { ion }\end{array}$ & $\mathrm{x}$ & & & & & $x$ & & \\
\hline Linear_regression & $x$ & & & & & $x$ & & $x$ \\
\hline Bayesian_linear_regression & $\frac{x}{x}$ & & & & & $\frac{x}{x}$ & & \\
\hline Neural_network_regression & $\frac{x}{x}$ & & & & & $\frac{x}{x}$ & $x$ & \\
\hline Decision_forest_regression & $\frac{x}{x}$ & & & & & $\frac{x}{x}$ & & $x$ \\
\hline $\begin{array}{l}\text { One_class_svM } \\
\text { On }\end{array}$ & & $x$ & & & & $\frac{x}{x}$ & & \\
\hline K_means & & & $x$ & & & & $x$ & \\
\hline Two-class_SVM & & & & $x$ & & $x$ & & \\
\hline $\begin{array}{l}\text { Two- } \\
\text { class_averaged_perceptron }\end{array}$ & & & & $x$ & & $x$ & & \\
\hline $\begin{array}{l}\text { Two- } \\
\text { class_Bayes_point_machine }\end{array}$ & & & & $x$ & & $x$ & & \\
\hline Two-class_decision_forest & & & & $x$ & & $x$ & & $x$ \\
\hline $\begin{array}{l}\text { Two- } \\
\text { class_logistic regression }\end{array}$ & & & & $x$ & & $x$ & & $x$ \\
\hline $\begin{array}{l}\text { Two- } \\
\text { class_boosted_decision_tree }\end{array}$ & & & & $x$ & & $\mathrm{x}$ & & $x$ \\
\hline Two-class_decision_jungle & & & & $x$ & & $x$ & & \\
\hline Two-class_neural_network & & & & $\hat{x}$ & & $\hat{x}$ & $x$ & \\
\hline $\begin{array}{l}\text { Multiclass_logistic_regressio } \\
\text { n }\end{array}$ & & & & & $x$ & $\mathrm{x}$ & & $x$ \\
\hline Multiclass_neural_network & & & & & $x$ & $x$ & $x$ & \\
\hline Multiclass_decision_jungle & & & & & $x$ & $x$ & & \\
\hline One-vs-all_multiclass & & & & & $x$ & $x$ & & \\
\hline $\begin{array}{l}\text { Boosted_decision_tree_regr } \\
\text { ession }\end{array}$ & $x$ & & & & & $x$ & & $x$ \\
\hline $\begin{array}{l}\text { PCA- } \\
\text { based_anomaly_detection }\end{array}$ & & $x$ & & & & $x$ & & $x$ \\
\hline Two-class_locally_deep_SVM & & & & $x$ & & $x$ & & \\
\hline Multiclass_decision_forest & & & & & $x$ & $x$ & & $x$ \\
\hline
\end{tabular}

As a second phase of validation, we run the approach using a bigger sampled dataset of 207 unique nodes and 95 instances against the same ontology. The application of the proposed approach achieves a maximum reduction of $70 \%$ lattice nodes, when RBindex threshold is $40 \%$. It is worth noting that the threshold is not the only reduction factor since RBindex will only be used when the first proposed reduction index (RAindex) cannot produce an unequivocal decision

As we are simplifying the lattice, part of the information is lost during the reduction stage. A very small dataset is more prone to lose crucial information yet this risk is reduced for realistic datasets with bigger sizes.

In this specific case, the sampled dataset did not have enough support to keep the general ontology concept ("prediction") in its position during the reduction process which caused the movement of this general concept to represent a more specialised concept in the resulted lattice ("Regression"/ "Predict Values") (shown in Fig. 4). However, when we did the same experiment using a larger sampled dataset, the same ontology concept kept in its position representing the general high-level ontology concept (even with a $90 \%$ reduction threshold).

As the ontology is manually created to capture knowledge about the domain whilst the sampled context is generated from real data that likely contains a wider range of properties and instances, the simplified lattice produced by our approach is expected to be similar but not necessarily identical to the domain ontology. One measure of the 
quality of the approach then would be to measure similarity - the percentage of the concepts from the ontology which appear in the final lattice. We would only consider ontology concepts which have instances represented in the formal context because the ontology could be much larger than the domain represented in the formal context.

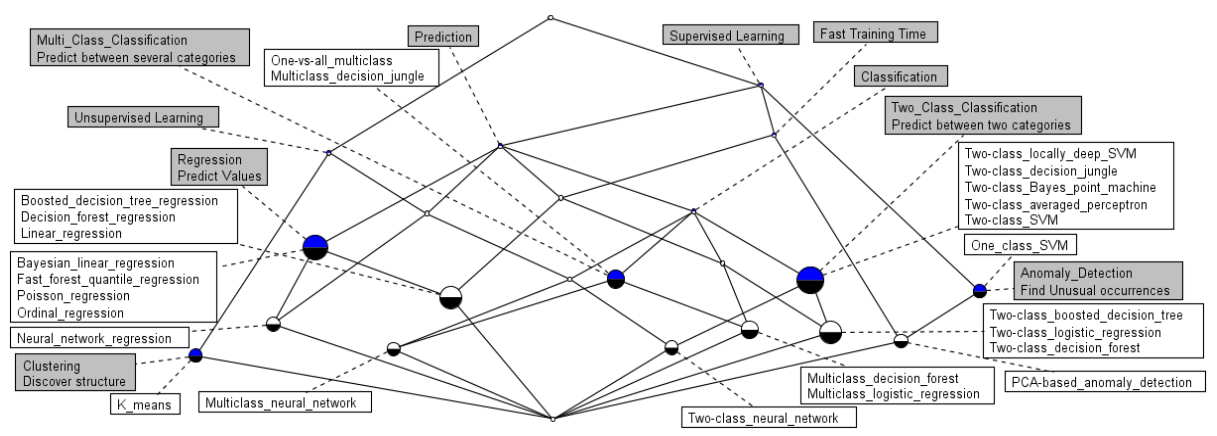

Fig. 3. The visualization of the extended FCA lattice resulted from the data alignment step.

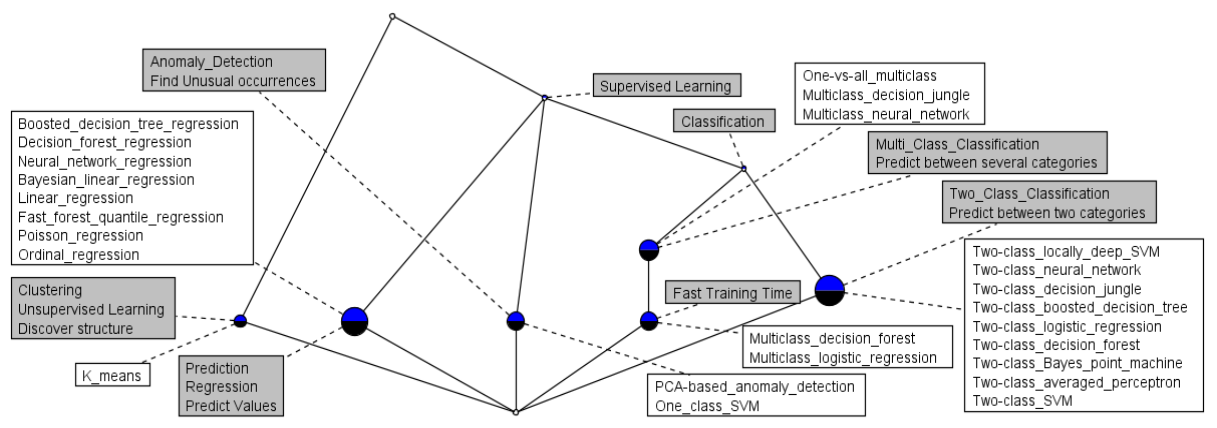

Fig. 4. The machine learning techniques lattice after applying the proposed approach (RBindex threshold of $40 \%$ ).

\section{Conclusion}

The proliferation of data and its importance for decision-making motivates the development of automatic ways of extracting information about relationships between data instances. Using Formal concept analysis (FCA) to achieve this does not yield simple enough lattices and requires a reduction step to generate structures which are simple to understand whilst preserving key information about the domain. Existing reduction algorithms are agnostic about any existing domain knowledge and this creates an opportunity to develop a new approach, which relies on the use of an existing domain knowledge encoded in a semantic ontology to inform the process of reducing the formal context and remove noisy data. Such an approach can enable the automatic processing of large data sets and thus support the construction of intelligent information systems. Our contribution to knowledge comprises an overall approach for ontology-driven lattice reduction and a novel index called the Discrimination Power Index (DPI). DPI is 
used to align the ontology-derived formal context with the sampled formal context, enabling the consequent use of the Ontology-informed Lattice Reduction indices and functions, which rely on the existing semantic knowledge encoded as ontology to guide the reduction process.

We report on two examples to confirm the feasibility and validity of the proposed approach. The approach achieves a significant reduction of lattice nodes creating a simplified, yet relevant, result that could be used in practice.

\section{References}

1. De Mauro, A., Greco, M. Grimaldi, M.: A formal definition of Big Data based on its essential features. Library Review 65(3), 122-135 (2016).

2. Singh, P. K., Aswani Kumar, C., Gani, A.: A comprehensive survey on formal concept analysis, its research trends and applications. International Journal of Applied Mathematics and Computer Science 26(2), 495-516 (2016).

3. Rouane, M. H., Huchard, M., Napoli, A., Valtchev, P.: A proposal for combining formal concept analysis and description logics for mining relational data. In: International Conference on Formal Concept Analysis, pp. 51-65, Springer, Berlin Heidelberg (2007).

4. Dias, S. M., Vieira, N. J.: Concept lattices reduction: definition, analysis and classification. Expert Systems with Applications 42(20), 7084-7097 (2015).

5. Stumme, G.: Using Ontologies and Formal Concept Analysis for Organizing Business Knowledge. In: Becker, J., Knackstedt, R. (eds.) Wissensmanagement mit Referenzmodellen 2002, pp. 163-174, Physica, Heidelberg (2002).

6. Sarmah, A. K., Hazarika, S. M., Sinha, S. K.: Formal concept analysis: current trends and directions. Artificial Intelligence Review 44(1), 47-86 (2015).

7. Ignatov, D. I.: Introduction to formal concept analysis and its applications in information retrieval and related fields. In: Braslavski, P., Karpov, N., Worring, M., Volkovich, Y., Ignatov, D. (eds.) Information Retrieval, vol. 505, pp. 42-141. Springer, Cham (2014).

8. Baader, F., Ganter, B., Sertkaya, B., Sattler, U.: Completing description logic knowledge bases using formal concept analysis. In Proceedings of the 20th International Joint Conference on Artificial Intelligence (IJCAI), pp. 230-235, Hyderabad, India (2007).

9. Ganter, B., Wille, R.: Formal concept analysis: mathematical foundations, Springer-Verlag, Berlin, 1999.

10. Gruber, T. R.: A Translation Approach to Portable Ontology Specification. Knowledge Acquisition 5, 199-220 (1993).

11. Lecue, F., Mehandjiev, N.: Seeking quality of web service composition in a semantic dimension. IEEE Transactions on Knowledge and Data Engineering, 23(6), 942-959 (2011).

12. Musen, M. A., Noy, N. F., Shah, N. H., Whetzel, P. L., Chute, C. G., Story, M. A., .., NCBO team.: The national center for biomedical ontology. Journal of the American Medical Informatics Association, 19(2), 190-195 (2011).

13. Bennett, M.: The financial industry business ontology: Best practice for big data. Journal of Banking Regulation, 14(3-4), 255-268 (2013).

14. Eriksson, O., Johannesson, P., Bergholtz, M.: Institutional ontology for conceptual modeling. Journal of Information Technology, 1-19 (2018).

15. Shah, T., Rabhi, F., Ray, P.: Investigating an ontology-based approach for Big Data analysis of inter-dependent medical and oral health conditions. Cluster Computing, 18(1), 351-367 (2015). 
16. De Giacomo, G., Lembo, D., Lenzerini, M., Poggi, A., Rosati, R.: Using ontologies for semantic data integration. In A Comprehensive Guide Through the Italian Database Research Over the Last 25 Years, pp. 187-202, Springer, Cham, 2018.

17. Singh, P. K., Kumar, C. A.: Concept lattice reduction using different subset of attributes as information granules. Granular computing 2(3), 159-173 (2017).

18. Li, J., Mei, C., Lv, Y.: Incomplete decision contexts: approximate concept construction, rule acquisition and knowledge reduction. International Journal of Approximate Reasoning 54(1), 149-165 (2013).

19. Wang, X., Zhang, W.: Attribute reduction in concept lattices based on deletion transformations. In $6^{\text {th }}$ International Conference on Natural Computation (ICNC), pp. 2065-2069, IEEE, Yantai, China, 2010.

20. Dias, S. M., Vieira, N. J.: Applying the JBOS reduction method for relevant knowledge extraction. Expert Systems with Applications 40(5), 1880-1887 (2013).

21. Kumar, C. A.: Fuzzy clustering-based formal concept analysis for association rules mining. Applied artificial intelligence 26(3), 274-301 (2012).

22. Zhang, S., Guo, P., Zhang, J., Wang, X., Pedrycz, W.: A completeness analysis of frequent weighted concept lattices and their algebraic properties. Data \& Knowledge Engineering 81, 104-117 (2012).

23. Belohlavek, R., Vychodil, V.: Formal concept analysis with background knowledge: attribute priorities. IEEE Transactions on Systems, Man, and Cybernetics, Part C (Applications and Reviews) 39(4), 399-409 (2009).

24. Bělohlávek, R., Sklenář, V., Zacpal, J.: Formal concept analysis with hierarchically ordered attributes. International Journal of General Systems 33(4), 383-394 (2004).

25. Sertkaya, B.: A survey on how description logic ontologies benefit from formal concept analysis. In Proceedings of the 7th International Conference on Concept Lattices and Their Applications, (CLA 2010), pp. 2-21, Sevilla, Spain, 2010.

26. Domenach, F., Portides, G.: Similarity Measures on Concept Lattices. In Wilhelm, A. Kestler, H. (eds.) Analysis of Large and Complex Data, pp. 159-169, Springer (2016).

27. Choi, S. S., Cha, S. H., Tappert, C. C.: A survey of binary similarity and distance measures. Journal of Systemics, Cybernetics and Informatics 8(1), 43-48 (2010).

28. Andrews, S., Polovina, S.: Exploring, Reasoning with and Validating Directed Graphs by Applying Formal Concept Analysis to Conceptual Graphs. In Graph Structures for Knowledge Representation and Reasoning (GKR 2017). Lecture Notes in Computer Science, pp. 3-28. Springer, Cham (2018).

29. Wirth, R. Hipp, J.: CRISP-DM: Towards a standard process model for data mining. In Proceedings of the 4th international conference on the practical applications of knowledge discovery and data mining, pp. 29-39, Manchester, UK, 2000.

30. Wolpert, D. H.: The Supervised Learning No-Free-Lunch Theorems. In Roy, R., Köppen, M., Ovaska, S., Furuhashi, T., Hoffmann, F. (eds.) Soft Computing and Industry, pp. 25-42, Springer, London, 2002.

31. Nural, M. V., Cotterell, M. E., Miller, J. A.: Using Semantics in Predictive Big Data Analytics. In IEEE International Congress on Big Data (BigData Congress), pp. 254-261, IEEE, New York, NY, USA, 2015.

32. Lin, M. S., Zhang, H., Yu, Z. G.: An ontology for supporting data mining process. In IMACS Multiconference on Computational Engineering in Systems Applications, pp. 2074-2077, IEEE, Beijing, China, 2006.

33. Azure Machine learning, [Online]. Available: https://docs.microsoft.com/en-us/azure/machine-learning/studio/algorithm-choice 